Relations industrielles

Industrial Relations

\title{
Women on the Job, by Judith b. Agassi, Lexington, Lexington Books, D.C. Heath and Company, 1979, 263 pp.
}

\section{Louise Turgeon}

Volume 35, numéro 2, 1980

URI : https://id.erudit.org/iderudit/029081ar

DOI : https://doi.org/10.7202/029081ar

Aller au sommaire du numéro

Éditeur(s)

Département des relations industrielles de l'Université Laval

ISSN

0034-379X (imprimé)

1703-8138 (numérique)

Découvrir la revue

Citer ce compte rendu

Turgeon, L. (1980). Compte rendu de [Women on the Job, by Judith b. Agassi, Lexington, Lexington Books, D.C. Heath and Company, 1979, 263 pp.] Relations industrielles / Industrial Relations, 35(2), 352-353.

https://doi.org/10.7202/029081ar

Tous droits réservés @ C Département des relations industrielles de l'Université Laval, 1980
Ce document est protégé par la loi sur le droit d'auteur. L’utilisation des services d'Érudit (y compris la reproduction) est assujettie à sa politique d'utilisation que vous pouvez consulter en ligne.

https://apropos.erudit.org/fr/usagers/politique-dutilisation/ 
L'auteur met aussi en relief l'effet négatif du corporatisme médical sur la concurrence au moyen du contingentement à l'entrée de la profession. On doit cependant regretter les intentions malicieuses qu'il prête à cet effet (en page 47) à un ex-président de l'Association Médicale Canadienne. Il me semble que les propos qui sont rapportés ne justifient pas l'interprétation que Blomqvist en donne.

Le deuxième chapitre présente une analyse comparative des systèmes américain, britannique et canadien. Tous ceux qui s'intéressent à l'économie de la santé reconnaîtront en ce chapitre un ajout important à la littérature existante.

À propos du système américain, où l'assurance privée prévaut, on apprend (p. 78) que le niveau de franchise adopté par les assurés est en général peu élevé alors même que des économies intéressantes pourraient être réalisées en optant pour un niveau de franchise plus élevé. Il semble donc que lorsque le consommateur a le choix, il a tendance à préférer une assurance intégrale. Le lecteur découvre aussi que la concurrence s'avère incapable de résoudre totalement le problème d'accessibilité aux services de santé puisque $5 \%$ de la population américaine ne détiendrait aucune forme d'assurance (p. 96).

À la page 126, Blomqvist fait grand état du fait que les Canadiens seraient de grands usagers des hôpitaux. Il nous signale que le séjour moyen à l'hôpital est de 11.3 jours au Canada, de 8.7 jours aux U.S.A. et de 12.8 jours en Grande-Bretagne (ces statistiques sont pour l'année 1974). Si on pousse l'analyse un peu plus loin, en utilisant l'information que nous livre Blomqvist à la page suivante, on constate alors que le coût moyen d'un séjour à l'hôpital est de $\$ 1085$ au Canada, de $\$ 1114$ aux U.S.A. et de $\$ 704$ en Grande-Bretagne. Il semble donc que la performance du système hospitalier canadien soutienne mieux la comparaison que ne le laisse entendre Blomqvist.

Il serait à propos de rappeler ici que la proportion du PNB consacré à la santé se situe en $1976 \mathrm{a} 6.8 \%$ au Canada et à $8.6 \%$ aux U.S.A. Remarquons de plus que le pourcen- tage canadien est à peu près stable depuis 1970 tandis que le pourcentage américain est en croissance continue.

Blomqvist termine son ouvrage par un chapitre consacré à diverses recommandations fortement inspirées de l'expérience américaine. Ces recommandations vont dans le sens d'une plus grande liberté de choix et d'un recours généralisé à la co-assurance ou à l'assurance avec franchise. Sous le régime préconisé par Blomqvist, le rôle de l'État serait essentiellement d'administrer un plan d'assurance convrant les exigences minimales a fixer par le législateur. Ce plan devrait s'autofinancer afin de concurrencer loyalement les assureurs privés appelés à jouer un rôle complémentaire.

À partir de l'information présentée par Blomqvist, il ressort que la situation des coûts de la santé apparaît davantage sous contrôle au Canada qu'aux U.S.A., en même temps que l'accessibilité aux services de santé est nettement meilleure au Canada. De plus, à partir des différentes statistiques que Blomqvist présente, il appert que l'état de santé du Canadien se compare avantageusement à celui de son voisin du sud. Bref, le recours à des changements drastiques, tels ceux préconisés par Blomqvist, ne me semble pas indiqué pour l'instant et je doute d'ailleurs que de tels changements soient désirés par la population.

\section{Denis MOFFET}

Université Laval

Women on the Job, by Judith B. Agassi, Lexington, Lexington Books, D.C. Heath and Company, 1979, $263 \mathrm{pp}$.

L'auteur, dans cette étude, rapporte les résultats d'une recherche empirique de taille qu'elle a effectuée auprès de quelque sept cent soixante (760) femmes occupant, sur le marché du travail, des emplois dans trois groupes occupationnels spécifiques soit le textile, le comme,ce au détail et le travail de bureau, et provenant de trois pays différents, à savoir les États-Unis, Israëxtile, le commerce au détail et le travail de bureau, et pro- 
venant de trois pays différents, à savoir les Etats-Unis, Israël et la république fédérale d'Allemagne.

Très bien documentée sur les théories et les recherches dans le domaine de la sociologie du travail, l'auteur s'est fixée comme objectif de vérifier la validité de deux groupes de ces théories. Elle examine tout d'abord les attitudes des femmes vis-à-vis leur travail et raffine sa recherche en analysant les facteurs d'influence de ces attitudes.

Judith B. Agassi pose bien son problème, fixe bien son objectif de travail et nous entraîne dans un cheminement dont les étapes sont d'une clarté et d'une méthode quasi cartésiennes. Après avoir assuré sa base théorique en relevant les études des Herzberg, Maslow, Blauner, Hulin, Levitin, Quinn, etc., elle s'attaque patiemment à la description et à l'analyse des statistiques pertinentes à sa population. Les données démographiques, celles de la tâche et du plan de carrière alliées à celles des attitudes au travail des répondantes, sont abondamment étoffées et constamment mises en corrélation les unes avec les autres.

C'est au quatrième chapitre que l'auteur intègre véritablement les théories sur les attitudes au travail qu'elle tente de vérifier. Elle en donne une définition nette, clarifie tous ses concepts, explique comment elle a cherché à les vérifier auprès de sa population étudiée pour nous faire ensuite part des conclusions auxquelles elle-même arrive, entérinant ou non celles déjà connues dans ce domaine des attitudes au travail et de leurs facteurs d'influence. C'est ainsi qu'elle tente de vérifier la théorie bien connue dite de la concordance (fit theory) ou celle de Herzberg et de Maslow sur les facteurs intrinsèques et extrinsisèques à la tâche par une série de corrélation entre différentes variables et les données de base de sa population.

Par ailleurs, Women on the Job prend toute son originalité dans sa seconde partie composée uniquement d'un ensemble de cent vingt-sept (127) tableaux illustrant statistiquement les données de bases, les variables d'attitudes et les corrélations que l'auteur a longuement étudiées dans la première partie de sa recherche.

Women on the Job est à recommander surtout à ceux et celles qui veulent raffiner leurs connaissances dans le domaine de la satisfaction au travail ou qui ont besoin de statistiques supplémentaires pour étayer leur propre cheminement dans cette sphère d'études.

Louise TURGEON

\section{Université Laval}

Les syndicats et l'OIT, Genève, Bureau international du travail, 1979, $103 \mathrm{pp}$.

Ce petit ouvrage n'a rien de scientifique. Il porte en sous-titre «manuel d'éducation ouvrière» et il réalise bien ce que ses auteurs se proposent: exposer aux travailleurs la nature et le fonctionnement de l'OIT, montrer comment les syndicats participent à ses conférences, réunions ou autres activités, mais aussi et surtout comment ils peuvent profiter de ses services.

Il est divisé en cinq chapitres de longueur et d'importance inégales: les syndicats et les origines de l'OIT; le tripartisme; le fonctionnement de l'OIT; l'action de l'OIT en faveur des travailleurs et des syndicats; quelques questions et réponses à propos de l'OIT. Quatre annexes fournissent: les dates importantes dans l'histoire de l'OIT; une bibliographie et des références; une nomenclature des textes fondamentaux de l'OIT pour les syndicalistes; et enfin la liste et adresses des bureaux extérieurs de l'OIT.

Comme il s'agit d'un instrument à caractère didactique, à la fin de chaque chapitre les auteurs ont ajouté des questions à discuter, des exercices de groupe ainsi que des sujets de recherches individuelles et collectives.

On y trouve, dans un langage accessible à tous, l'essentiel de ce qu'il faut savoir sur l'OIT. Cet ouvrage peut être utile à un public qui dépasse largement celui des travailleurs syndiqués.

Université Laval

Gérard DION 